\title{
Bevacizumab for refractory solid tumors in pediatric patients
}

\author{
Martin Benesch ${ }^{1 \dagger}$ \& \\ Christian Urban ${ }^{2}$ \\ ${ }^{\dagger}$ Author for correspondence \\ ${ }^{1}$ Medical University of Graz, \\ Division of Pediatric \\ Hematology \& Oncology, \\ Department of Pediatrics \& \\ Adolescent Medicine, \\ Auenbruggerplatz 30, \\ A-8036 Graz, Austria \\ Tel.: +4331638580427; \\ Fax: +433163853450 ; \\ E-mail:martin.benesch@ \\ klinikum-graz.at \\ ${ }^{2}$ Tel.: +433163853485; \\ Fax: +433163853450 ; \\ E-mail: christian.urban@ \\ meduni-graz.at
}

\begin{abstract}
Evaluation of: Bender JL, Adamson PC, Reid JM et al.: Phase I trial and pharmacokinetic study of bevacizumab in pediatric patients with refractory solid tumors: a Children's Oncology Group study. J. Clin. Oncol. 26, 399-405

(2008). Angiogenesis is important for both tumor growth and development of metastases. Vascular endothelial growth factor is a potent proangiogenic factor. Bevacizumab, a humanized antibody against vascular endothelial growth factor is currently being tested for various malignancies among adult cancer patients, but has not yet been prospectively evaluated in children and young adults with malignant solid tumors. The results of the first Phase I trial of bevacizumab in this population are here presented by Bender et al. Bevacizumab administered at 5,10 and $15 \mathrm{mg} / \mathrm{kg}$ doses every 2 weeks was well tolerated with no major toxicities (i.e., hemorrhage or thrombosis) observed. None of the patients achieved an objective response. Trials assessing combinations of bevacizumab with cytotoxic chemotherapy or other targeted therapies are warranted to further explore the efficacy of this drug in childhood malignancies.
\end{abstract}

\section{Summary of methods \& results}

Bender $e t a l$. present the results of a dose-escalating Phase I trial of bevacizumab in pediatric patients with refractory solid tumors [1]. Enrollment of children and young adults with hematological or oncological diseases in prospective clinical trials has resulted in a dramatic improvement of prognosis during the past 40 years. Survival at 5 years increased from 54\% in patients diagnosed between 1978 and 1982 to $75 \%$ in patients diagnosed between 1993 and 1997 [2]. This is at least partly explained by the fact that the up-front therapy in pediatric cancer patients is very well standardized. However, the introduction of new compounds for pediatric cancer patients is often delayed for a number of reasons. The study by Bender $e t$ al. is the first to evaluate pharmacokinetic, toxicity and response data in children and young adults suffering from different solid tumors refractory to standard antineoplastic therapy. The starting dose of bevacizumab was $5 \mathrm{mg} / \mathrm{kg}$. Drug dosage was increased to 10 and $15 \mathrm{mg} / \mathrm{kg}$ within subsequent patient cohorts. One course was defined as two administrations of bevacizumab separated by a 2-week interval. Patients could receive a maximum of 24 courses. The protocol included a detailed evaluation of efficacy and side effects, as well as pharmacokinetic and pharmacodynamic studies. The results of this study are summarized in this paper.

\section{Patient characteristics}

A total of 21 patients were enrolled, of whom 19 received (at least one dose of) bevacizumab. Median patient age at study entry was 13 years (range: 1 to 21 years).

Ewing sarcoma was the most common diagnosis $(\mathrm{n}=5)$, followed by osteosarcoma $(\mathrm{n}=3)$, neuroblastoma $\quad(\mathrm{n}=2), \quad$ nephroblastoma $(\mathrm{n}=2)$, hepatoblastoma $(\mathrm{n}=2)$ and other malignant solid tumors $(n=5)$. Patients with lymphoma, CNS metastases or primary brain tumors were excluded.

\section{Toxicity}

A total of three patients each were assessable for toxicity at the 5 and $10 \mathrm{mg} / \mathrm{kg}$ dose level; a total of twelve patients could be evaluated at the maximum dose level of $15 \mathrm{mg} / \mathrm{kg}$. Overall, these 18 patients received a total of 67 courses (median number of courses: three; maximum number of courses: 16). No dose-limiting toxicity was observed in any of these cases. A maximum tolerated dose was not defined. Hemorrhagic or thrombotic events did not occur. Although blood pressures increased from day 1 to day 21 in 11 out of 16 patients, this increase of blood pressure did not result in hypertension-related symptoms.

\section{Efficacy}

None of the patients achieved a complete or partial response. 


\section{Pharmacokinetics \& pharmacodynamics}

Half-life $\left(\mathrm{t}_{1 / 2}\right)$ varied considerably, particularly at the $15 \mathrm{mg} / \mathrm{kg}$ dose level. Median $\mathrm{t}_{1 / 2}$ was 12.8 days (range: 9.9 to 12.9 days) among the three patients evaluated at the $5 \mathrm{mg} / \mathrm{kg}$ dose level. Corresponding figures for patients receiving $15 \mathrm{mg} / \mathrm{kg}$ (five patients were assessed) were 11.7 days (range: 4.4 to 14.6 ). Of note, novel parameters (i.e., the number of circulating endothelial cells [CEC] and their subpopulations), which might be able to predict and monitor antiangiogenic treatment, were also analyzed. Although the findings are comparable to those obtained in adults, no definite conclusions can be drawn at this time.

\section{Discussion}

Preclinical data suggest that antiangiogenic therapy might be effective in malignant nonepithelial tumors of childhood [3]. However, until recently studies evaluating antiangiogenic compounds such as bevacizumab in the pediatric population were lacking. We recently presented our experiences with the compassionate use of bevacizumab in 15 patients (male: $\mathrm{n}=8$; female: $\mathrm{n}=7$; median age: 14.6 years) with recurrent or progressive solid tumors [4]. Two patients had benign vascular tumors; treatment with bevacizumab was initiated in these two patients due to the potentially life-threatening site of the tumor. Based on data derived from adult cancer patients, we used bevacizumab at $5-10 \mathrm{mg} / \mathrm{kg}$ every 2-3 weeks. All patients with malignant solid tumors were extensively pretreated and received cytotoxic chemotherapy in addition to bevacizumab. Duration of bevacizumab therapy ranged from 1.5 to 23 months, respectively. Radiographic objective responses (partial responses) were observed in two of our patients with astrocytoma grade III and in one patient each with neuroblastoma and pleomorphic rhabdomyosarcoma, respectively. Bevacizumab was well tolerated, even in patients who received this drug over a prolonged period of time. However, we did not study growth- or development-related toxicities in our patients in particular.

The present study by Bender $e t$ al., which provided prospectively generated data on safety, efficacy and pharmacokinetics of bevacizumab is an important contribution for pediatric oncologists. To a remarkable extent, the clinical investigation of new drugs in children and adolescents is hampered due to increasing safety concerns [5]. So far, the toxicity profile of antiangiogenic drugs such as bevacizumab is very different from that of the known cytotoxic drugs [6]. Although the presently available clinical experiences suggest that bevacizumab is well tolerated in the majority of patients, some of the adult patients treated with bevacizumab will develop serious side effects, such as cardiovascular or thromboembolic events, bleeding or gastrointestinal perforation [6,7]. In addition, other side effects, such as hypertension or proteinuria, are reported to occur in up to $30-40 \%$ of patients [6]. The pathophysiological mechanisms of side effects related to antiangiogenic treatment are not fully elucidated [6]. So far, severe treatment-related side effects were not observed in the two recently presented series including children and young adults $[1,4]$. However, since the number of children and adolescents receiving treatment with bevacizumab will continue to grow, one might expect that the number of reported toxicities among the pediatric population will also increase in the future. Data on longterm side effects of bevacizumab are limited. With the current strategy to use bevacizumab mainly in patients with metastatic disease, only a few of these patients will survive long enough to develop treatment-related late effects. With further improvement in long-term survival of patients receiving antiangiogenic treatment, questions regarding long-term safety will become more prominent. Thus, long-term toxicity should be carefully and prospectively evaluated, particularly in children and young adults who are planned to receive bevacizumab.

Although preclinical [3] and clinical studies [8,9] have demonstrated that bevacizumab monotherapy inhibits tumor growth, the antitumor activity of bevacizumab is best enhanced by combining it with cytotoxic chemotherapy. So far, ongoing clinical trials are almost exclusively testing the combination of bevacizumab with either cytotoxic chemotherapy or other targeted therapies [7-8,10-12]. Since antiangiogenic drug therapy alone is probably insufficient to induce tumor regression in extensively pretreated cancer patients, not unexpectedly no complete or partial response was observed in the trial by Bender et al. One might assume that combination therapies including bevacizumab will produce higher response rates compared with monotherapy in pediatric cancer patients.

\section{Executive summary \& future perspective}

The study by Bender $e t a l$. is the first prospective trial to evaluate bevacizumab in pediatric cancer patients. The acute safety profile of bevacizumab appears to be reasonably good with no major 


\section{Bevacizumab in pediatric patients - PRIORITY PAPER EVALUATION}

toxicity reported so far. Analysis of biological markers to assess efficacy of antiangiogenic therapy will certainly be extended in future clinical trials. The results presented by Bender et al. will hopefully serve as the basis for the initiation of Phase II trials evaluating combinations of bevacizumab with conventional chemotherapy. This could expand our therapeutic options in children and young adults with recurrent or progressive malignant solid tumors in the near future.

\section{Financial \& competing interests disclosure}

The authors have no relevant affiliations or financial involvement with any organization or entity with a financial interest in or financial conflict with the subject matter or materials discussed in the manuscript. This includes employment, consultancies, honoraria, stock ownership or options, expert testimony, grants or patents received or pending, or royalties.

No writing assistance was utilized in the production of this manuscript.

\section{Bibliography}

1. Bender JL, Adamson PC, Reid JM et al: Phase I trial and pharmacokinetic study of bevacizumab in pediatric patients with refractory solid tumors: a Children's Oncology Group study. J. Clin. Oncol. 26(3), 399-405 (2008).

2. Magnani C, Pastore G, Coebergh JW et al: The trends in survival after childhood cancer in Europe, 1978-1997: report from the Automated Childhood Cancer Information System project (ACCIS). Eur. J. Cancer 42(13), 1981-2005 (2006)

3. Gerber HP, Ferrara N. Pharmacology and pharmacodynamics of bevacizumab as monotherapy or in combination with cytotoxic therapy in preclinical studies. Cancer Res. 65(3), 671-680 (2005).

4. Benesch M, Windelberg M, Sauseng W et al: : Compassionate use of bevacizumab (Avastin ${ }^{\circledR}$ ) in children and young adults with refractory or recurrent solid tumors.
Ann. Oncol. doi: 10.1093/annonc/mdm510 (2007) (Epub ahead of print).

5. Paolucci P, Jones KP, del Carmen Cano Garcinuno M, Catapano M, Iolascon A, Ceci A: Challenges in prescribing drugs for children with cancer. Lancet Oncol. 9(2), 176-183 (2008).

6. Eskens FA, Verweij J: The clinical toxicity profile of vascular endothelial growth factor (VEGF) and vascular endothelial growth factor receptor (VEGFR) targeting angiogenesis inhibitors; a review. Eur. J. Cancer 42(18), 3127-3139 (2006).

7. Hurwitz H, Fehrenbacher L, Novotny W et al: Bevacizumab plus irinotecan, fluorouracil, and leucovorin for metastatic colorectal cancer. N. Engl. J. Med. 350(23), 2335-2342 (2004).

8. Rini BI: Vascular endothelial growth factortargeted therapy in renal cell carcinoma: current status and future directions. Clin. Cancer. Res. 13(4), 1098-1106 (2007).
9. Cobleigh MA, Langmuir VK, Sledge GW et al: : A Phase I/II dose-escalation trial of bevacizumab in previously treated metastatic breast cancer. Semin. Oncol. 30(5 Suppl. 16), 117-124 (2003).

10. Rodriguez J, Zarate R, Bandres E et al: Combing chemotherapy and targeted therapies in metastatic colorectal cancer. World J. Gastroenterol. 13(44), 5867-5876 (2007).

11. Miller KD, Chap LI, Holmes FA et al:: Randomized Phase III trial of capecitabine compared with bevacizumab plus capecitabine in patients with metastatic breast cancer. J. Clin. Oncol. 23, 792-799 (2005).

12. Crane CH, Ellis LM, Abbruzzese JL et al: Phase I trial evaluating the safety of bevacizumab with concurrent radiotherapy and capecitabine in locally advanced pancreatic cancer. J. Clin. Oncol. 24, 1145-1151 (2006). 